\title{
The new era of risk reclassification in cardiovascular imaging
}

\author{
Leslee J. Shaw, PhD, FASNC, FACC, FAHA
}

Although for decades, risk stratification and prognostic modeling have been the focus of many clinical registries in nuclear cardiology; more recent adaptations to the traditional clinical outcomes approach has been presented. One of these concepts is the idea of risk reclassification where by prognostic models are compared in terms of newly identified low and high risk patients. ${ }^{1,2}$ This concept has clinical appeal in that the calculation of the net reclassification improvement (NRI) identifies the fraction of patients that are uniquely defined as low risk and conversely uniquely defined as high risk. Thus, as we evaluate the role of nuclear cardiology above and beyond clinical or exercise test results, the NRI can be an invaluable means to quantify improvement in risk detection.

Recently, a report noted that treadmill results using the Duke treadmill score improved risk reclassification in only $11.2 \%$ of patients when compared to clinical pretest risk estimates. ${ }^{3}$ The NRI was 0.112 which means that improved risk re-classification was only documented in only $\sim 1$ in 10 patients. When compared to nuclear imaging results, the NRI was 0.358 revealing that nearly 1 in 3 patients had improved risk reclassification when compared to treadmill testing and clinical pretest risk estimates.

Although this is one of the first NRI reports in symptomatic patient cohorts, data in asymptomatic individuals report an NRI of 0.25 for coronary calcium scoring when compared to the Framingham risk score. ${ }^{4}$ Thus, the NRI can provide interesting insight into the added contribution of $\mathrm{CV}$ imaging in varied patient and population cohorts.

One may envision that as more data is published on the NRI that a league table of evidence may aid in defining optimal utilization for CV imaging. For example, the NRI for coronary calcium and high sensitivity

From the Emory Clinical Cardiovascular Research Institute, Emory University School of Medicine, Atlanta, GA.

Reprint requests: Leslee J. Shaw, PhD, FASNC, FACC, FAHA, Emory Clinical Cardiovascular Research Institute, Emory University School of Medicine, Atlanta, GA; lshaw3@emory.edu.

J Nucl Cardiol 2011;18:536-7.

1071-3581/\$34.00

Copyright $@ 2011$ American Society of Nuclear Cardiology.

doi:10.1007/s12350-011-9387-7
C-reactive protein was 0.254 and $0.0565,{ }^{5}$ respectively, compared to the Framingham Risk Score. This example nicely illustrates the difference between a risk factor (i.e., inflammation) as compared to an imaging marker that is a direct marker of atherosclerosis (i.e., calcification). Using this same approach, data comparing the NRI for stress echocardiography as compared to stress myocardial perfusion imaging may prove invaluable as we enter into the era of the development of multimodality appropriate use criteria.

Moreover, as we embark on comparative effectiveness research, use of the NRI can be an important means to compare the effectiveness of various imaging modalities in terms of improvement in prognostic modeling. If one considers a comparison of the NRI for wall motion when compared to perfusion imaging results, it is interesting to speculate where an improved reclassification may be observed. Perfusion imaging results may have a better NRI for improvements in low risk findings when compared to wall motion results due to an improved detection of intermediate stenoses. Moreover, improvements in the detection of high risk individuals may be observed in coronary artery disease patients with moderate-severe ischemia in the estimation of ischemic-type events.

An advantage to the NRI is its clinical appeal in documenting the proportion of patients that are aided by refining risk estimates and this can form the basis for defining appropriateness of testing and guiding healthcare coverage policies within a given clinical indication. It remains an important societal discussion to compare the economic value of higher cost tests that may have higher NRI with lower cost tests with lower NRI calculations. Conversely, the NRI can be a valuable means to guide new technology assessments. Moreover, in this era of limited financial means in our healthcare system, the use of NRI across varied patient populations can provide support to differentiate the effectiveness of nuclear cardiology and a defining metric of quality to define appropriate utilization.

\section{References}

1. Pencina MJ, D'Agostino RB Sr, D'Agostino RB Jr, Vasan RS. Evaluating the added predictive ability of a new marker: From area under the ROC curve to reclassification and beyond. Stat Med 2008;27:157-72. (discussion 207-12). 
2. Pencina MJ, D'Agostino RB Sr, Steyerberg EW. Extensions of net reclassification improvement calculations to measure usefulness of new biomarkers. Stat Med 2011;30:11-21.

3. Shaw LJ, Wilson PW, Hachamovitch R, Hendel RC, Borges-Neto $\mathrm{S}$, Berman DS. Improved near-term coronary artery disease risk classification with gated stress myocardial perfusion SPECT. JACC Cardiovasc Imaging 2010;3:1139-48.
4. Polonsky TS, McClelland RL, Jorgensen NW, et al. Coronary artery calcium score and risk classification for coronary heart disease prediction. JAMA 2010;303:1610-6.

5. Wilson PW, Pencina M, Jacques P, Selhub J, D'Agostino R Sr, O'Donnell CJ. C-reactive protein and reclassification of cardiovascular risk in the Framingham Heart Study. Circ Cardiovasc Qual Outcomes 2008;1:92-7. 